\title{
Molecular gas and dark neutral medium in the outskirts of Chamaeleon
}

\author{
H. Liszt ${ }^{1}$, M. Gerin ${ }^{2}$, and I. Grenier ${ }^{3}$ \\ ${ }^{1}$ National Radio Astronomy Observatory, 520 Edgemont Road, Charlottesville, VA 22903, USA \\ e-mail: hliszt@nrao.edu \\ ${ }^{2}$ LERMA, Observatoire de Paris, PSL Research University, CNRS Sorbonne Universités, UPMC Univ. Paris 06, École Normale \\ Supérieure, 75005 Paris, France \\ e-mail: maryvonne.gerin@ens. fr \\ 3 AIM, CEA-IRFU/CNRS/Université Paris Diderot, Département d'Astrophysique, CEA, Saclay, 91191 Gif-sur-Yvette, France \\ e-mail: isabelle.grenier@cea.fr
}

Received 5 April 2018 / Accepted 2 July 2018

\begin{abstract}
Context. More gas is inferred to be present in molecular cloud complexes than can be accounted for by $\mathrm{H} \mathrm{I}$ and $\mathrm{CO}$ emission, a phenomenon known as dark neutral medium (DNM) or CO-dark gas for the molecular part.

Aims. We aim to investigate whether molecular gas can be detected in Chamaeleon where gas column densities in the DNM were inferred and $\mathrm{CO}$ emission was not detected.

Methods. We took $\lambda 3 \mathrm{~mm}$ absorption profiles of $\mathrm{HCO}^{+}$and other molecules toward 13 background quasars across the Chamaeleon complex, only one of which had detectable $\mathrm{CO}$ emission. We derived the $\mathrm{H}_{2}$ column density assuming $\mathrm{N}\left(\mathrm{HCO}^{+}\right) / \mathrm{N}\left(\mathrm{H}_{2}\right)=3 \times 10^{-9}$ as before.

Results. With the possible exception of one weak continuum target, $\mathrm{HCO}^{+}$absorption was detected in all directions, $\mathrm{C}_{2} \mathrm{H}$ in eight directions and $\mathrm{HCN}$ in four directions. The sightlines divide into two groups according to their DNM content, with one group of eight directions having $\mathrm{N}(\mathrm{DNM}) \gtrsim 2 \times 10^{20} \mathrm{~cm}^{-2}$ and another group of five directions having $\mathrm{N}(\mathrm{DNM})<0.5 \times 10^{20} \mathrm{~cm}^{-2}$. The groups have comparable mean N(H I) associated with Chamaeleon $6-7 \times 10^{20} \mathrm{~cm}^{-2}$ and total hydrogen column density per unit reddening $6-7 \times 10^{21} \mathrm{~cm}^{-2} \mathrm{mag}^{-1}$. They differ, however, in having quite different mean reddening $0.33 \mathrm{vs} .0 .18 \mathrm{mag}$, mean N(DNM) $3.3 \mathrm{vs}$. $0.14 \times 10^{20} \mathrm{~cm}^{-2}$ and mean molecular column density $2 \mathrm{~N}\left(\mathrm{H}_{2}\right)=5.6$ vs. $0.8 \times 10^{20} \mathrm{~cm}^{-2}$. The gas at more positive velocities is enriched in molecules and DNM.

Conclusions. Overall the quantity of $\mathrm{H}_{2}$ inferred from $\mathrm{HCO}^{+}$can fully account for the previously inferred DNM along the sightlines studied here. $\mathrm{H}_{2}$ is concentrated in the high-DNM group, where the molecular fraction is $46 \%$ vs. $13 \%$ otherwise and $38 \%$ overall. Thus, neutral gas in the outskirts of the complex is mostly atomic but the DNM is mostly molecular. Saturation of the H I emission line profile may occur along three of the four sightlines having the largest DNM column densities, but there is no substantial reservoir of "dark" atomic or molecular gas that remains undetected as part of the inventory of dark neutral medium.
\end{abstract}

Key words. ISM: clouds - ISM: molecules - ISM: structure - ISM: atoms

\section{Introduction}

The use of $\lambda 21 \mathrm{~cm} \mathrm{H} \mathrm{I} \mathrm{and} \lambda 2.6 \mathrm{~mm} \mathrm{CO}$ emission to trace the column densities of atomic and molecular hydrogen is fundamental to study of the interstellar medium (ISM). Yet, it is commonly the case that a combination of these two tracers fails to account for all of the gas that is inferred to be present from other tracers of the total column density, for instance the Fermi $\gamma$-ray flux (Grenier et al. 2005) and the Planck sub-mm dust optical depth (Planck Collaboration Int. XXVIII 2015). This has led to the concept of "dark" gas (Grenier et al. 2005) which could, in principle and depending on particular circumstances, be attributed either to saturation of the $\mathrm{H}$ I emission profile (Fukui et al. 2015; Okamoto et al. 2017) or to the inability of CO emission to track the $\mathrm{H}_{2}$ column density at intermediate reddening (Wolfire et al. 2010; Planck Collaboration Int. XXVIII 2015; Remy et al. 2018) where the column density of $\mathrm{H}_{2}$ is appreciable but $\mathrm{N}(\mathrm{CO})$ is less than $10^{15} \mathrm{~cm}^{-2}$ and the integrated $\mathrm{CO}$ brightness $W_{\mathrm{CO}}$ is below $1 \mathrm{~K}-\mathrm{km} \mathrm{s}^{-1}$ (Liszt 2017). Donate \& Magnani (2017) argue that the CO-dark gas inventory in Pegasus-Pisces is halved by their extensive new detections of $\mathrm{CO}$ emission at levels $W_{\mathrm{CO}}<1 \mathrm{~K}-\mathrm{km} \mathrm{s}^{-1}$.

A flexible approach to determining the origin of the missing gas is to express the total gas column density $\mathrm{N}(\mathrm{H})$ as a linear combination of $\mathrm{N}(\mathrm{H} \mathrm{I}) \propto \mathrm{W}_{\mathrm{HI}}$ and $\mathrm{N}\left(\mathrm{H}_{2}\right) \propto W_{\mathrm{CO}}$ as represented by the $\mathrm{H} \mathrm{I}$ and $\mathrm{CO}$ emission profile integrals $\mathrm{W}_{\mathrm{HI}}$ and $W_{\mathrm{CO}}$, but with an added term N(DNM) for the so-called dark neutral medium that is absent in emission and could be in either or both atomic and molecular form (Planck Collaboration Int. XXVIII 2015; Remy et al. 2018). By comparing the linear combination $\mathrm{N}(\mathrm{H})_{\mathrm{emp}}=\mathrm{N}(\mathrm{H} \mathrm{I})+\mathrm{N}(\mathrm{DNM})+2 \mathrm{~N}\left(\mathrm{H}_{2}\right)$ with the map of a (total) column density tracer such as the gamma-ray flux or dust opacity, the spatial distribution of the DNM can be derived over an entire cloud complex, thereby associating the DNM with the distribution of the $\mathrm{H} \mathrm{I}$ and $\mathrm{CO}$ tracers and with the structure of the cloud complex itself.

In this work we take advantage of an existing analysis of the DNM in the Chamaeleon cloud complex (Planck Collaboration Int. XXVIII 2015) to compare DNM and molecular gas, but with an $\mathrm{H}_{2}$ tracer that is sensitive to very 
Table 1. Sightline and spectral line properties.

\begin{tabular}{|c|c|c|c|c|c|c|c|c|c|c|}
\hline Source & $\begin{array}{c}\alpha(\mathrm{J} 2000) \\
\text { hh.mmssss }\end{array}$ & $\begin{array}{c}\delta(\mathrm{J} 2000) \\
\text { dd.mmssss } \\
\end{array}$ & $\begin{array}{c}l \\
\left({ }^{\circ}\right) \\
\end{array}$ & $\begin{array}{c}b \\
\left({ }^{\circ}\right) \\
\end{array}$ & $\begin{array}{l}E_{B-V^{a}} \\
(\mathrm{mag})\end{array}$ & $\begin{array}{c}\mathrm{N}(\mathrm{H} \mathrm{I})^{b} \\
\left(10^{20} \mathrm{~cm}^{-2}\right) \\
\end{array}$ & $\begin{array}{c}S_{89.2} \\
(\mathrm{Jy})\end{array}$ & $\sigma_{l / c^{c}}$ & $\begin{array}{c}W_{\mathrm{HCO}^{+}}{ }^{d} \\
\left(\mathrm{~km} \mathrm{~s}^{-1}\right) \\
\end{array}$ & $\begin{array}{r}W_{\mathrm{HCN}^{e}} \\
\left(\mathrm{~km} \mathrm{~s}^{-1}\right) \\
\end{array}$ \\
\hline J0942-7731 & 09.424275 & -77.311158 & 293.321 & -18.329 & 0.33 & 9.1 & 0.203 & 0.0400 & $1.142(0.067)^{f}$ & $<0.155$ \\
\hline J1058-8003 & 10.584331 & -80.035416 & 298.010 & -18.288 & 0.15 & 6.0 & 1.189 & 0.0067 & $0.201(0.009)$ & $<0.032$ \\
\hline J1136-6827 & 11.360210 & -68.270609 & 296.070 & -6.590 & 0.47 & 21.7 & 0.466 & 0.0195 & $1.241(0.035)$ & $0.293(0.008)$ \\
\hline J1145-6954 & 11.455362 & -69.540179 & 297.316 & -7.747 & 0.38 & 16.8 & 0.537 & 0.0188 & $0.870(0.031)$ & $0.150(0.007)$ \\
\hline J1147-6753 & 11.473340 & -67.534176 & 296.958 & -5.767 & 0.30 & 23.0 & 1.552 & 0.0064 & $0.054(0.010)$ & $<0.034$ \\
\hline J1152-8344 & 11.525322 & -83.440943 & 301.238 & -21.058 & 0.28 & 9.8 & 0.113 & 0.0719 & $0.240(0.070)$ & $<0.342$ \\
\hline J1224-8313 & 12.245438 & -83.131010 & 302.095 & -20.391 & 0.26 & 8.7 & 0.300 & 0.0249 & $0.945(0.044)$ & $0.345(0.040)$ \\
\hline B1251-7138 & 12.545983 & -71.381840 & 303.213 & -8.769 & 0.28 & 17.0 & 0.574 & 0.0166 & $0.139(0.027)$ & $<0.091$ \\
\hline J1312-7724 & 13.123874 & -77.241306 & 304.122 & -14.582 & 0.46 & 11.0 & 0.207 & 0.0390 & $0.271(0.036)$ & $<0.202$ \\
\hline J1550-8258 & 15.505916 & -82.580650 & 308.272 & -22.047 & 0.11 & 6.3 & 0.352 & 0.0261 & $0.242(0.040)$ & $<0.158$ \\
\hline J1617-7717 & 16.174928 & -77.171846 & 313.426 & -18.854 & 0.09 & 6.2 & 2.464 & 0.0044 & $0.059(0.007)$ & $<0.022$ \\
\hline J1723-7713 & 17.235085 & -77.135020 & 315.688 & -21.800 & 0.26 & 7.7 & 0.309 & 0.0322 & $1.507(0.052)$ & $0.952(0.030)$ \\
\hline J1733-7935 & 17.334070 & -79.355537 & 313.606 & -23.268 & 0.14 & 7.3 & 0.597 & 0.0149 & $0.057(0.020)$ & $<0.078$ \\
\hline
\end{tabular}

Notes. ${ }^{(a)}$ Schlegel et al. (1998). ${ }^{(b)} \mathrm{N}(\mathrm{H} \mathrm{I})=\int T_{B} \mathrm{~d} v \times 1.823 \times 10^{18} \mathrm{~cm}^{-2}$ from the Gass III H I profile (Kalberla \& Haud 2015). ${ }^{(c)}$ Line/continuum rms at $89.2 \mathrm{GHz}$ at zero optical depth. ${ }^{(d)} \mathrm{N}\left(\mathrm{HCO}^{+}\right)=1.10 \times 10^{12} \mathrm{~cm}^{-2} \mathrm{~W}_{\mathrm{HCO}^{+}}{ }^{(e)} \mathrm{N}(\mathrm{HCN})=1.89 \times 10^{12} \mathrm{~cm}^{-2} \mathrm{~W}_{\mathrm{HCN}}$. Upper limits are $3 \sigma$. (f) Quantities in parenthesis are the standard deviation.

weakly excited molecular gas and to molecular gas having smaller column density than is typically sampled in $\mathrm{CO}$ emission: we observed $\mathrm{HCO}^{+}$and other strongly-polar species in absorption at $\lambda 3 \mathrm{~mm}$ toward 13 relatively strong background continuum sources seen by chance against the outskirts of the Chamaeleon complex where $\mathrm{CO}$ had been detected in only one direction with $W_{\mathrm{CO}}=2.4 \mathrm{~K}-\mathrm{km} \mathrm{s}^{-1}$, only slightly above the detection limit.

In this work we discuss the implications of our detection of molecular absorption in all of the directions lacking observable $\mathrm{CO}$ emission over wide areas of the outskirts of the Chamaeleon cloud complex. Section 2 presents the new observations, summarizes existing observational material that is cited and the underlying assumptions that undergird our work. In Sect. 3 we discuss the new results and Sect. 4 is a summary.

\section{Observations and data reduction}

\subsection{New ALMA absorption measurements and $\mathrm{H}_{2}$ column densities}

We observed the $J=1-0$ lines of $\mathrm{HCO}^{+}, \mathrm{HCN}$ and $\mathrm{C}_{2} \mathrm{H}$ in absorption toward the 13 continuum sources listed in Table 1, whose locations on the sky are shown in Fig. 1. CS $J=2-1$ and $\mathrm{H}^{13} \mathrm{CO}^{+}$were observed but not detected and are not discussed in the text below. The work was conducted under ALMA Cycle 4 project 2016.1.00714.S whose pipeline data products were delivered in 2017 February and March. Spectra were extracted from the continuum-subtracted pipeline-processed data cubes at the pixel of peak continuum flux in the continuum map made from the spectral window containing each line, and divided by the continuum flux in the continuum map for that spectral window at that pixel. Fluxes at $89.2 \mathrm{GHz}$ ranged from $0.11 \mathrm{Jy}$ for $\mathrm{J} 1152$ to $2.5 \mathrm{Jy}$ for $\mathrm{J} 1617$ as noted in Table 1 . Each spectrum consisted of 1919 semi-independent channels spaced $61 \mathrm{kHz}$ corresponding to $0.205 \mathrm{~km} \mathrm{~s}^{-1}$ at the $89.189 \mathrm{GHz}$ rest frequency of $\mathrm{HCO}^{+}$. Absorption from $\mathrm{CS}$ and $\mathrm{H}^{13} \mathrm{CO}^{+}$was not detected and will not be further discussed.

$\mathrm{H}_{2}$ column densities $\mathrm{N}\left(\mathrm{H}_{2}\right)$ are derived from the absorption line profile of $\mathrm{HCO}^{+}$using $\mathrm{N}\left(\mathrm{HCO}^{+}\right)=1.10 \times 10^{12} \mathrm{~cm}^{-2} \int \tau \mathrm{d} v$ with the profile integral in units of $\mathrm{km} \mathrm{s}^{-1}$ (Ando et al. 2016;
Gerin \& Liszt 2017). $\mathrm{N}\left(\mathrm{HCO}^{+}\right)$is derived under the assumption that the rotational excitation temperature is that of the cosmic microwave background, which is justified by the weakness of $\mathrm{HCO}^{+}$emission in diffuse molecular gas, even when the absorption is quite optically thick (Liszt 2012; Liszt \& Pety 2016). We also assume $\mathrm{N}\left(\mathrm{HCO}^{+}\right) / \mathrm{N}\left(\mathrm{H}_{2}\right)=3 \times 10^{-9}$. The relative abundance $\mathrm{N}\left(\mathrm{HCO}^{+}\right) / \mathrm{N}\left(\mathrm{H}_{2}\right)$ can be derived in various ways; most direct is to refer it to the relative abundances of $\mathrm{OH}$ and $\mathrm{CH}$ (Liszt \& Lucas 1996; Liszt \& Gerin 2016), both of which are directly determined optically, eg (Weselak et al. 2010), and give the same value for $\mathrm{N}\left(\mathrm{HCO}^{+}\right) / \mathrm{N}\left(\mathrm{H}_{2}\right)$.

\subsection{H I column densities}

H I column densities were derived for all directions from Galactic All-Sky Survey GASS III $\lambda 21 \mathrm{~cm} \mathrm{H} \mathrm{I} \mathrm{emission} \mathrm{spectra}$ (Kalberla \& Haud 2015). Table 1 shows total column densities $\mathrm{N}(\mathrm{H} \mathrm{I})$ derived from the line brightness profile integral over all velocities in the optically thin limit,

$\left.N(\mathrm{H} \mathrm{I})\right|_{\text {tot }}=1.823 \times 10^{18} \mathrm{~cm}^{-2} \int T_{B} \mathrm{~d} v$.

Column densities of H I associated with the Chamaeleon complex, N(H I) $\left.\right|_{\text {cham }}$, are given in Table 2 . These were derived following the methodology described by Planck Collaboration Int. XXVIII (2015) and Remy et al. (2018). GASS III H I emission profiles were decomposed into pseudo-Voigt profiles (Gaussian cores with the possibility of co-centered Lorentzian wings) and the profile integrals of all components centered within the velocity range of the Chamaeleon complex were summed, again in the optically thin limit that provided the best fit in the analysis of Planck Collaboration Int. XXVIII (2015).

\section{3. $D N M$}

Maps of the column density of dark neutral medium - the component that is not fully visible in $\mathrm{H} \mathrm{I}$ or $\mathrm{CO}$ emission - were published for most of the sightlines by Planck Collaboration Int. XXVIII (2015). Specific values of N(DNM) along the present sightlines were extracted from that work for the present use, along with values toward J1136 and J1147 that were just outside 


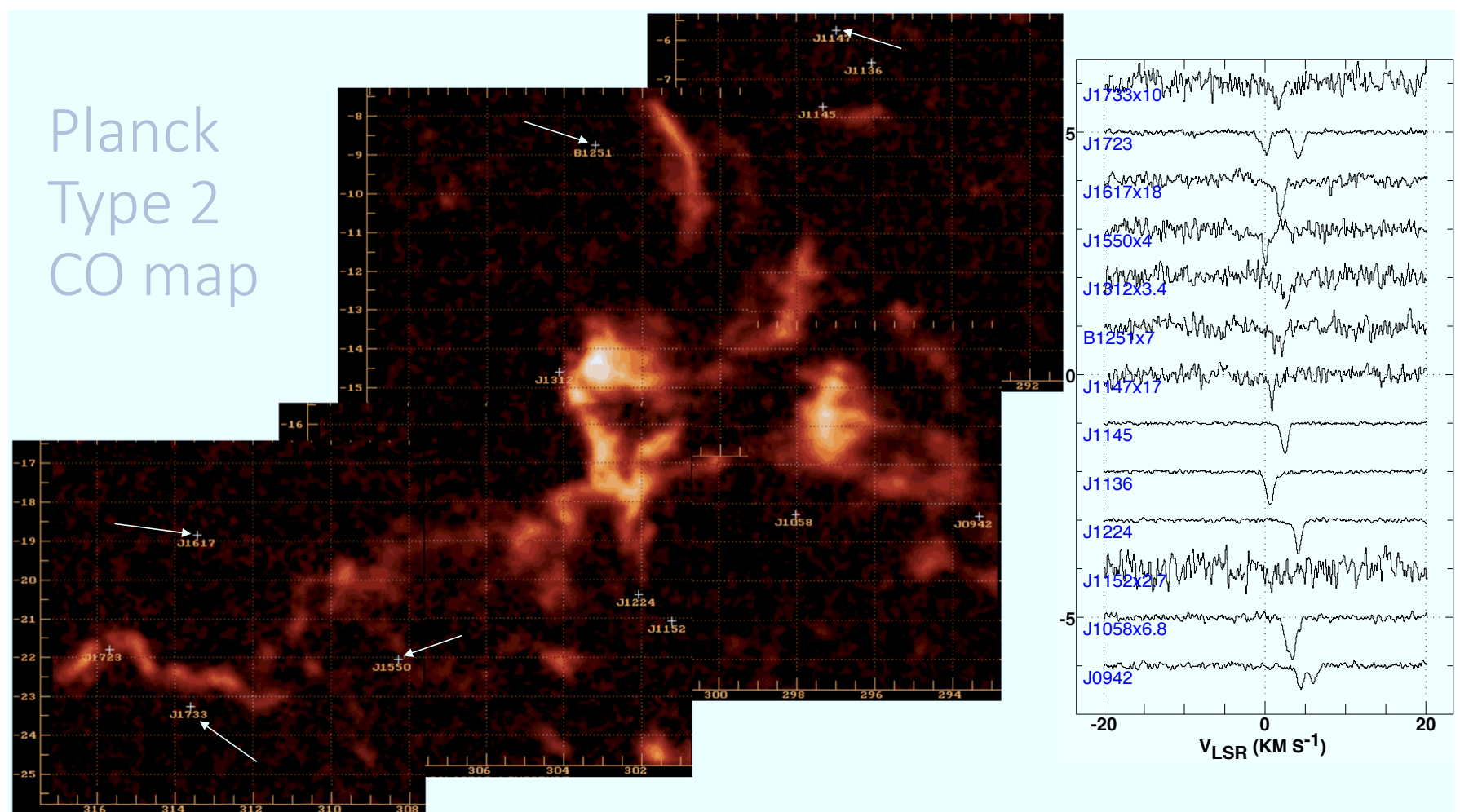

Fig. 1. Left panel: the positions of the background sources observed here are projected against a mosaic of cutouts of Planck Type 2 integrated

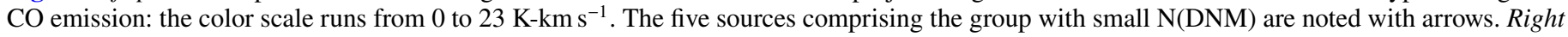
panel: the $\mathrm{HCO}^{+}$absorption profiles in all directions, shifted vertically and scaled up in some cases.

Table 2. Target by target sightline gas and dust properties in descending N(DNM) order.

\begin{tabular}{|c|c|c|c|c|c|c|c|c|}
\hline $\begin{array}{l}\mathrm{N}(\mathrm{DNM}) \\
\left(10^{20} \mathrm{~cm}^{-2}\right)\end{array}$ & Source & $\begin{array}{l}E_{B-V} \\
(\mathrm{mag})\end{array}$ & $\begin{array}{l}\left.\mathrm{N}(\mathrm{H} \mathrm{I})\right|_{\mathrm{tot}}{ }^{a} \\
\left(10^{20} \mathrm{~cm}^{-2}\right)\end{array}$ & $\begin{array}{l}\left.\mathrm{N}(\mathrm{H} \mathrm{I})\right|_{\mathrm{cham}}{ }^{b} \\
\left(10^{20} \mathrm{~cm}^{-2}\right)\end{array}$ & $\begin{array}{c}\mathrm{N}(\mathrm{DNM})^{c} \\
\left(10^{20} \mathrm{~cm}^{-2}\right)\end{array}$ & $\begin{array}{c}2 \mathrm{~N}\left(\mathrm{H}_{2}\right)^{d} \\
\left(10^{20} \mathrm{~cm}^{-2}\right)\end{array}$ & $\begin{array}{c}\mathrm{N}(\mathrm{H}) / E_{B-V}{ }^{e} \\
\left(10^{21} \mathrm{~cm}^{-2} \mathrm{mag}^{-1}\right)\end{array}$ & $\begin{array}{c}\mathrm{N}\left(\mathrm{H}_{2}\right) / X_{\mathrm{CO}}^{0}{ }^{-1} \\
\left(\mathrm{~K}-\mathrm{km} \mathrm{s}^{-1}\right)\end{array}$ \\
\hline \multirow[t]{9}{*}{$\gtrsim 2$} & $\mathrm{~J} 1152^{g}$ & 0.28 & 9.8 & 5.05 & 5.15 & 1.76 & 4.1 & 0.5 \\
\hline & J1312 & 0.46 & 11.0 & 6.71 & 3.96 & 1.98 & 2.8 & 0.5 \\
\hline & $\mathrm{J} 0942^{g}$ & 0.33 & 9.1 & 6.17 & 3.82 & 8.20 & 5.2 & 2.0 \\
\hline & $\mathrm{J} 1058$ & 0.15 & 6.0 & 4.70 & 3.71 & 1.50 & 5.0 & 0.4 \\
\hline & $\mathrm{J} 1224^{g, h}$ & 0.26 & 8.7 & 5.63 & 3.35 & 7.20 & 6.1 & 1.8 \\
\hline & $\mathrm{J} 1145^{g, h}$ & 0.38 & 16.8 & 9.98 & 2.23 & 6.20 & 6.1 & 1.5 \\
\hline & $\mathrm{J} 1723^{g, h, i}$ & 0.26 & 7.7 & 5.00 & 1.95 & 11.1 & 7.2 & 2.8 \\
\hline & $\mathrm{J} 1136^{g, h}$ & 0.47 & 21.7 & 9.15 & 1.72 & 9.1 & 7.0 & 1.6 \\
\hline & $\operatorname{mean}(\sigma)$ & $0.33(0.11)$ & $12.7(6.3)$ & $6.6(2.0)$ & $3.3(1.3)$ & $5.6(3.6)$ & $5.4(1.4)$ & $1.4(0.9)$ \\
\hline \multirow[t]{6}{*}{$\lesssim 0.5$} & $\mathrm{~J} 1617$ & 0.09 & 6.2 & 4.31 & 0.45 & 0.43 & 7.3 & 0.1 \\
\hline & $\mathrm{J} 1550$ & 0.11 & 6.3 & 4.16 & 0.27 & 1.90 & 7.4 & 0.5 \\
\hline & $\mathrm{J} 1147$ & 0.30 & 23.0 & 7.73 & 0.00 & 0.40 & 7.8 & 0.1 \\
\hline & $\mathrm{B} 1251^{g}$ & 0.28 & 17.0 & 6.60 & 0.00 & 0.84 & 6.0 & 0.2 \\
\hline & $\mathrm{J} 1733^{g}$ & 0.14 & 7.3 & 5.25 & 0.00 & 0.58 & 5.2 & 0.2 \\
\hline & $\operatorname{mean}(\sigma)$ & $0.18(0.10)$ & $12.7(8.6)$ & $5.6(1.5)$ & $0.14(0.21)$ & $0.83(0.62)$ & $6.7(1.1)$ & $0.22(0.16)$ \\
\hline
\end{tabular}

Notes. ${ }^{(a)}$ As in Table 1. $\left.{ }^{(b)} \mathrm{N}(\mathrm{H}$ I $)\right|_{\text {cham }}$ is $\mathrm{N}(\mathrm{H} \mathrm{I})$ associated with the Chamaeleon complex. ${ }^{(c)} \mathrm{N}(\mathrm{DNM})$ from Planck Collaboration Int. XXVIII (2015). ${ }^{(d)} \mathrm{N}\left(\mathrm{H}_{2}\right)=\mathrm{N}\left(\mathrm{HCO}^{+}\right) / 3 \times 10^{-9} .{ }^{(e)} \mathrm{N}(\mathrm{H})=2 \mathrm{~N}\left(\mathrm{H}_{2}\right)+\left.\mathrm{N}(\mathrm{H} \mathrm{I})\right|_{\text {tot }} .{ }^{(f)}$ The predicted integrated $\mathrm{CO} J=1-0$ brightness $\left(W_{\mathrm{CO}}\right)$ for $\mathrm{X}_{\mathrm{CO}}=$ $2 \times 10^{20} \mathrm{H}_{2} \mathrm{~cm}^{-2}\left(\mathrm{~K}_{\mathrm{km} \mathrm{s}}{ }^{-1}\right)^{-1}$. ${ }^{(g)} \mathrm{C}_{2} \mathrm{H}$ detected. ${ }^{(h)} \mathrm{HCN}$ detected. ${ }^{(i)} W_{\mathrm{CO}}=2.4 \mathrm{~K}-\mathrm{km} \mathrm{s}^{-1}$.

the region considered by Planck Collaboration Int. XXVIII (2015). for their interstellar fit but still in the analysis region. All of these values are given in Table 2. N(DNM) is comparatively small in five directions and no DNM was needed along three of these: a determination of $\mathrm{N}(\mathrm{DMN})$ was made along all sightlines including those for which 0 -values are given in Table 2.

\subsection{CO emission}

The derivation of N(DNM) by Planck Collaboration Int. XXVIII (2015) used NANTEN maps of $J=1-0$ CO emission from Mizuno et al. (2001). The CO emission map in Fig. 1 uses the Planck Type $2 \mathrm{CO}$ product which is virtually indistinguishable 
from the NANTEN map in a blink comparison. The rms of the Planck Type $2 \mathrm{CO}$ product over the fields comprising Fig. 1 is $\Delta W_{\mathrm{CO}}=0.45 \pm 0.05 \mathrm{~K}-\mathrm{km} \mathrm{s}^{-1}$, leading to a detection limit comparable to that of the NANTEN maps, about $2 \mathrm{~K}-\mathrm{km} \mathrm{s}^{-1}$ (Mizuno et al. 2001). It should be noted that the Planck CO maps have been hashed over small regions in the immediate vicinity of several of our sightlines, presumably as the result of point-source removal, something of a Catch-22 situation for the present study.

\subsection{Reddening and dust optical depth}

The $6^{\prime}$ resolution dust-emission maps scaled to optical reddening $E_{B-V}$ by Schlegel et al. (1998) are cited in Tables 1 and 2. Those reddening values can be converted to Planck $353 \mathrm{GHz}$ dust optical depth using the relationship established by Planck Collaboration XI (2014) between the $353 \mathrm{GHz}$ dust optical depth and the $E_{B-V}$ values of Schlegel et al. (1998), $E_{B-V} / \tau_{353}=(1.49 \pm 0.03) \times 10^{4} \mathrm{mag}$.

\subsection{Conventions}

Velocities presented with the spectra are taken with respect to the kinematic definition of the local standard of rest. $N(H)$ is the column density of $\mathrm{H}$-nuclei in neutral atomic and molecular form, $\mathrm{N}(\mathrm{H})=\mathrm{N}(\mathrm{H} \mathrm{I})+2 \mathrm{~N}\left(\mathrm{H}_{2}\right)$.

\section{Molecules and dark neutral medium in the Chamaeleon complex}

The use of H I, CO, Planck sub-mm dust and Fermi $\gamma$ ray emission observed on $4^{\prime}-16^{\prime}$ and larger angular scales to derive gas properties across the Chamaeleon complex was discussed by Planck Collaboration Int. XXVIII (2015) and further refined by Remy et al. (2018). This work adds the information gained from molecular absorption profiles observed along 13 sightlines toward point-like extragalactic mm-wave continuum sources that otherwise serve as phase calibrators for the ALMA telescope.

The angular resolution of interferometric absorption line observations is the apparent angular size of the background source in the presence of interstellar scattering, which is milliarcseconds at radio wavelengths: the instrumental resolution, approximately $1^{\prime \prime}$ during the observing, does not determine this. Broader-beam emission observations do more spatial averaging, but both types of observations do comparable sampling of the gas along the line of sight which is the more important aspect. Differences between emission and absorption line profiles generally reflect the character of the gas distribution, not the differing spatial resolution that was employed to obtain them (Clark 1965).

This work takes advantage of the fact that $\mathrm{HCO}^{+}$absorption is more widespread than CO emission (Lucas \& Liszt 1996; Liszt \& Lucas 1998; Liszt \& Pety 2012), presumably because $\mathrm{N}(\mathrm{CO})$ varies so sharply with $\mathrm{N}\left(\mathrm{H}_{2}\right)$ and $E_{B-V}$ (Burgh et al. 2007; Sonnentrucker et al. 2007; Sheffer et al. 2008) while $\mathrm{N}\left(\mathrm{HCO}^{+}\right) / \mathrm{N}\left(\mathrm{H}_{2}\right)$ can be taken as constant. The net result is that $\mathrm{HCO}^{+}$absorption is the more reliable tracer of $\mathrm{H}_{2}$ along the lines of sight observed here and even very extreme differences in angular resolution may introduce scatter, but do not introduce bias. To accommodate this, we derive and tabulate the properties of the gas along the individual sightlines (Tables 1 and 2), as we must, but the discussion is cast in terms of the average properties of two groups and the differences between them. Discussion of individual sightlines is largely left for the concluding parts of the discussion and the final figure.

\subsection{General properties}

General properties of the sightlines studied here are given in Table 1, with entries as explained in Sect. 2. A finding chart is presented in Fig. 1, where the locations of the continuum targets are shown against a background image of the Planck Type 2 CO map and the 5 sightlines having small column densities $\mathrm{N}(\mathrm{DNM})<0.5 \times 10^{20} \mathrm{~cm}^{-2}$ are noted. All of the $\mathrm{HCO}^{+}$absorption profiles are shown in right panel in Fig. 1, in some cases scaled up for easier viewing: they are shown again in Fig. 2 with the NANTEN CO profiles (see Sect. 3.2).

The continuum sources sample the borders of the CO emission distribution and avoid the regions of detected $\mathrm{CO}$ emission, except for J1723. A recent search of the ALMA calibrator database for newly discovered strong continuum background targets in Chamaeleon was not fruitful. Unless weaker continuum sources are used, the current survey represents the state of the art in this part of the sky.

In Table 2 the sources are reordered by descending N(DNM), and grouped according to whether $\mathrm{N}(\mathrm{DNM}) \gtrsim 2 \times 10^{20} \mathrm{H} \mathrm{cm}^{-2}$ (eight sources) or $\mathrm{N}(\mathrm{DNM})<0.5 \times 10^{20} \mathrm{H} \mathrm{cm}^{-2}$ (five sources). The sightlines with smaller N(DNM) are called out in Fig. 1 where it is seen that they occur across the entire length of the Chamaeleon cloud complex. This is noteworthy, given the limited kinematic range of the molecular absorption in these directions, see Sect. 3.2.

The two groups have similar mean total $\mathrm{H}$ I column density $\left\langle\left.\mathrm{N}(\mathrm{H} \mathrm{I})\right|_{\text {tot }}\right\rangle=13 \times 10^{20} \mathrm{~cm}^{-2}$ averaged over all sightlines in each group, similar mean $\mathrm{N}(\mathrm{H}$ I) associated with Chamaeleon $\left\langle\left.\mathrm{N}(\mathrm{H} \mathrm{I})\right|_{\text {cham }}\right\rangle=5.6-6.6 \times 10^{20} \mathrm{~cm}^{-2}$ and similar mean total column density per unit reddening $\left\langle\mathrm{N}(\mathrm{H}) / E_{B-V}\right\rangle \approx 5.4-6.7 \times 10^{21} \mathrm{~cm}^{-2}(\mathrm{mag})^{-1}$, but the group of eight DNM-rich sightlines has much larger mean reddening $\left\langle E_{B-V}\right\rangle$ (0.33 vs. $\left.0.18 \mathrm{mag}\right)$, mean DNM column density $\langle\mathrm{N}(\mathrm{DNM})\rangle\left(3.3\right.$ vs. $\left.0.14 \times 10^{20} \mathrm{~cm}^{-2}\right)$ and column density of $\mathrm{H}$-nuclei in molecular form $\left\langle 2 \mathrm{~N}\left(\mathrm{H}_{2}\right)\right\rangle\left(5.6\right.$ vs. $\left.0.83 \times 10^{20} \mathrm{~cm}^{-2}\right)$. Their similarity in terms of the total column density per unit reddening is only possible because of the larger contribution of $\mathrm{H}_{2}$ in the group with higher mean reddening.

\subsection{Kinematics and abundance}

The kinematic signature of the difference between the two groups is illustrated in Fig. 3 where the mean H I profiles for each group (left panel) and their difference (right panel) are plotted along with the mean $\mathrm{HCO}^{+}$optical depth profile, suitably scaled. The figure makes the point that the difference in $\mathrm{H} \mathrm{I}$, an increase, occurs at 2-8 $\mathrm{km} \mathrm{s}^{-1}$ where most of the molecular gas is found. The low-velocity component of $\mathrm{HCO}^{+}$at $v \approx 0.5 \mathrm{~km} \mathrm{~s}^{-1}$ is quite widespread, being present toward J1136, J1147, J1550 and J1723 and is present in both DNM-groups.

This point is further elaborated in Fig. 4, comparing the mean $\mathrm{HCO}^{+}$optical depth profiles in the two groups. $\mathrm{HCO}^{+}$absorption in the group with lower N(DNM) is of course weaker, but it also occurs only at $v \lesssim 2 \mathrm{~km} \mathrm{~s}^{-1}$. As shown in Fig. 1, sightlines with small N(DNM) span the breadth of the Chamaeleon complex and in principle could sample the full range of velocities, but they do not. This suggests that the higher molecular abundance and increased kinematic complexity in the higher DNM group are associated, as, for instance, when $\mathrm{H}_{2}$ production is enhanced in the presence of slow shocks associated with turbulent flows (Micic et al. 2012; Valdivia et al. 2017)

Figure 5 compares the mean optical depth profiles of $\mathrm{HCO}^{+}$, $\mathrm{HCN}$ and $\mathrm{C}_{2} \mathrm{H}$. The $\mathrm{HCN}-\mathrm{HCO}^{+}$relationship is monotonic in the sense that the weakest and strongest components are the 


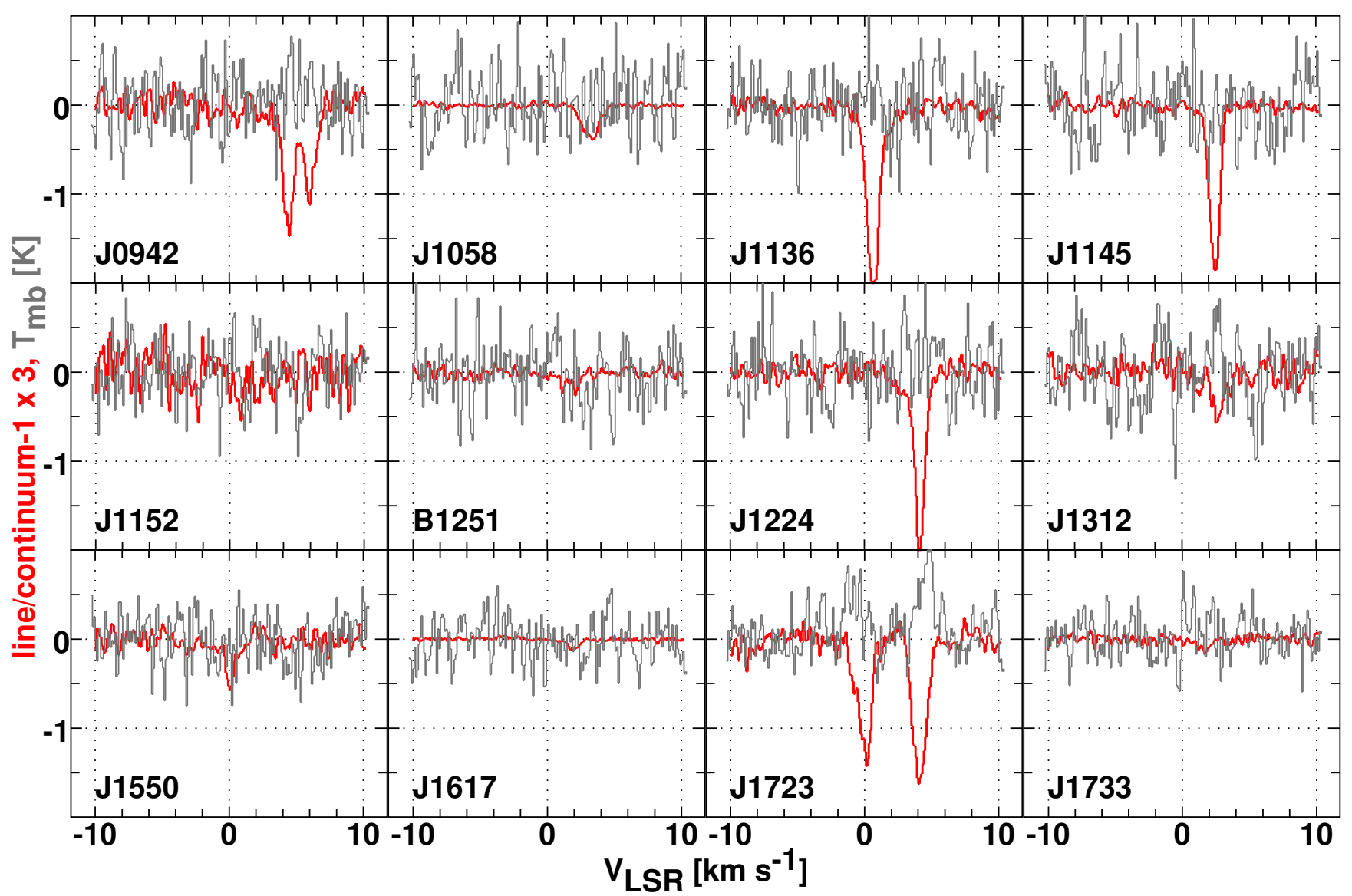

Fig. 2. Comparison of NANTEN CO emission (black) and $\mathrm{ALMA} \mathrm{HCO}^{+}$absorption (red) profiles in the directions observed. The CO emission is shown on the main-beam scale of the NANTEN telescope in Kelvins and the $\mathrm{HCO}^{+}$absorption profiles have been scaled up by a factor three. $\mathrm{CO}$ was reliably detected only toward J1723 and not observed toward J1147 (not shown here).

same in both species but the $\mathrm{HCN} / \mathrm{HCO}^{+}$ratio varies and is noticeably larger in the strongest component at $4 \mathrm{~km} \mathrm{~s}^{-1}$. This mimics the $\mathrm{N}(\mathrm{HCN})-\mathrm{N}\left(\mathrm{HCO}^{+}\right)$relationship that is seen along individual lines of sight where there is a rapid increase of $\mathrm{N}(\mathrm{HCN})$ at $\mathrm{N}\left(\mathrm{HCO}^{+}\right) \gtrsim 10^{12} \mathrm{~cm}^{-2}$ (Liszt \& Lucas 2001; Ando et al. 2016; Riquelme et al. 2018; Liszt \& Gerin 2018) or $\mathrm{N}\left(\mathrm{H}_{2}\right) \gtrsim 3 \times 10^{20} \mathrm{~cm}^{-2}$. $\mathrm{N}(\mathrm{HCN})$ and $\mathrm{N}(\mathrm{CN})$ are seen to vary in fixed proportion and $\mathrm{CN}$ is well known to increase in abundance for $\mathrm{N}\left(\mathrm{H}_{2}\right) \gtrsim 3 \times 10^{20} \mathrm{~cm}^{-2}$ in optical spectroscopy of the diffuse molecular gas (Sheffer et al. 2008). Although $\mathrm{C}_{2} \mathrm{H}$ absorption is faint, the $\mathrm{C}_{2} \mathrm{H}$ and $\mathrm{HCO}^{+}$line profiles look fairly similar. $\mathrm{C}_{2} \mathrm{H}$ is known to be present in the diffuse molecular gas in quasi-constant abundance ratio relative to $\mathrm{CH}$ and to $\mathrm{HCO}^{+}$. The present data are consistent with the established relation between $\mathrm{HCO}^{+}$and $\mathrm{C}_{2} \mathrm{H}$ (Lucas \& Liszt 2000; Ando et al. 2016; Riquelme et al. 2018; Liszt \& Gerin 2018).

\subsection{Balance among $\mathrm{N}\left(\mathrm{H}_{2}\right), N(D N M)$ and $N(\mathrm{HI})$}

Overall, the hydrogen in the outskirts of the Chamaeleon complex is mainly in atomic form. In ascending order, averaged over all sightlines, $\langle\mathrm{N}(\mathrm{DNM})\rangle=2.1 \times 10^{20} \mathrm{~cm}^{-2}$, $2 \mathrm{~N}\left(\mathrm{H}_{2}\right)=3.8 \times 10^{20} \mathrm{~cm}^{-2}\left\langle\left.\mathrm{~N}(\mathrm{H} \mathrm{I})\right|_{\text {cham }}\right\rangle=6.2 \times 10^{20} \mathrm{~cm}^{-2}$ and this ordering applies to most of the sightlines individually as well. There is only one direction (J1152) where $\mathrm{N}(\mathrm{DNM})$ even slightly exceeds $\left.\mathrm{N}(\mathrm{H} \mathrm{I})\right|_{\text {cham }}$ and only three where $2 \mathrm{~N}\left(\mathrm{H}_{2}\right)>\left.\mathrm{N}(\mathrm{H} \mathrm{I})\right|_{\text {cham }}$. The point is that the molecular component must represent a minority fraction of the total hydrogen even if all the DNM turns out to be $\mathrm{H}_{2}$. As summarized in Table $3, \mathrm{H}_{2}$ comprises $46 \%$ of the H-nuclei in the group having more DNM, but only $13 \%$ otherwise and $36 \%$, about one-third, in total. Overall, the molecular/atomic balance of the outlying Chamaeleon gas is very similar to that of the diffuse ISM as a whole, with estimates of the hydrogen fraction in $\mathrm{H}_{2}$ ranging from $25 \%$ to 40\% (Savage et al. 1977; Liszt \& Lucas 2002).

\subsection{CO emission, observed and expected}

The NANTEN CO profiles toward our targets are shown and compared with the ALMA $\mathrm{HCO}^{+}$absorption profiles in Fig. 2, except toward $\mathrm{J} 1147$ that was not covered in the CO mapping. CO emission was reliably detected only toward $\mathrm{J} 1723$ where the $\mathrm{CO}$ and $\mathrm{HCO}^{+}$lines coincide in velocity and $W_{\mathrm{CO}}=2.4 \mathrm{~K}-\mathrm{km} \mathrm{s}^{-1}$. The CO profile toward J0942 is suggestive, given the overlap with $\mathrm{HCO}^{+}$absorption, but other equally strong and apparently spurious features are present in the CO spectrum. Weak spurious signals in consecutive channels are seen in many $\mathrm{CO}$ spectra. For example, toward $\mathrm{J} 1312$ there are three equally strong positivegoing features, only one of which coincides with the $\mathrm{HCO}^{+}$ absorption, and three comparably strong negative-going features. Moreover, the $\mathrm{HCO}^{+}$absorption toward $\mathrm{J} 1312$ is too weak to support a credible CO detection, as is also the case for J1152. For $\mathrm{J} 1224$, a weak positive-going $\mathrm{CO}$ feature near 0-velocity is too far removed from the $\mathrm{HCO}^{+}$absorption to be reliable.

A null CO signal, $W_{\mathrm{CO}}=0$, was therefore taken in all directions except J1723 in the succeeding discussion. In this regard, 


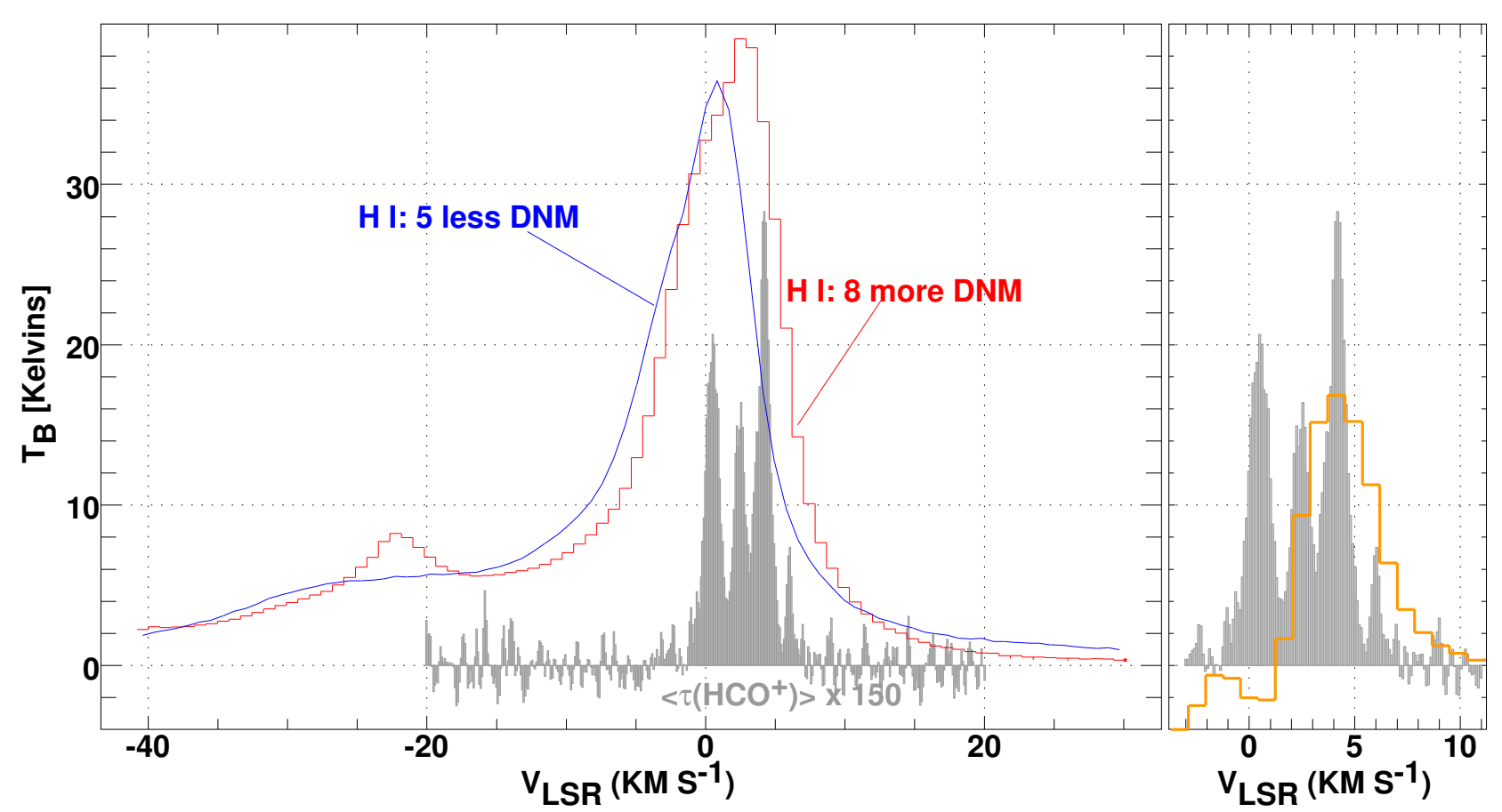

Fig. 3. Kinematics of the H I, separated by N(DNM). Left panel: shown separately are the mean H I profiles for the two groups of sources according to their $\mathrm{N}(\mathrm{DNM})$ : H I emission in the group having more DNM is plotted as a red histogram and emission in the other group is shown as a blue line. Also shown is the mean $\mathrm{HCO}^{+}$optical depth profile for all sources scaled upward by a factor 150. Right panel: as in left panel but the $\mathrm{H}$ I profile plotted in orange is the difference of the H I profiles shown in left panel. The greatest difference between the H I profiles coincides with the peak of the $\mathrm{HCO}^{+}$optical depth.

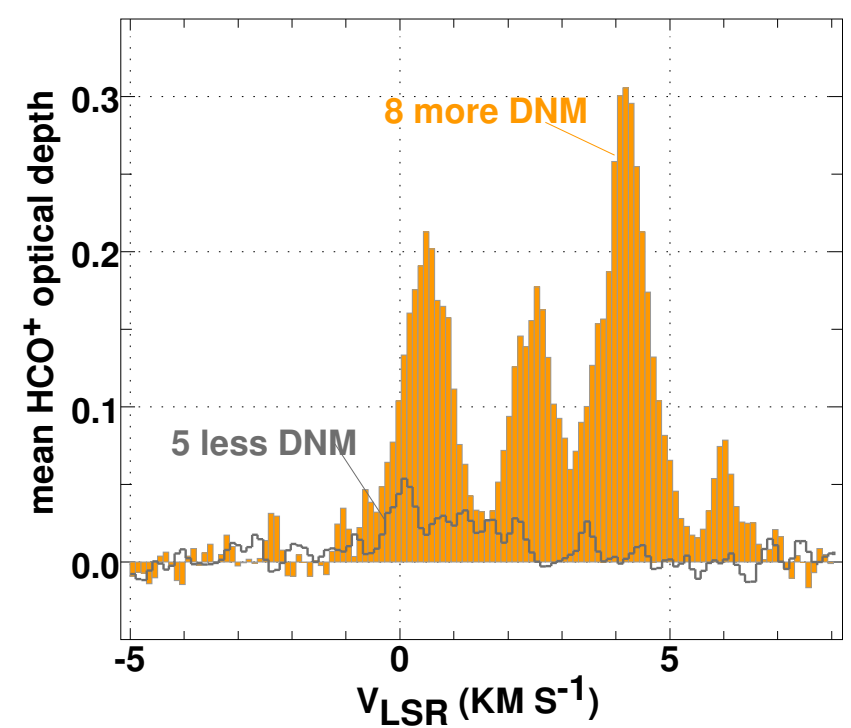

Fig. 4. Kinematics of the molecular gas traced by $\mathrm{HCO}^{+}$, separated by $\mathrm{N}(\mathrm{DNM})$. Shown separately are the average $\mathrm{HCO}^{+}$optical depths of the five sources with weak NDNM (dark gray histogram) and the eight sources with stronger $\mathrm{N}(\mathrm{DNM})$ (orange bars).

note that we hedged this assumption with an accepted ALMA Cycle 5 proposal to search for CO absorption so that our assumption of null detections will be tested. Perhaps more importantly, the main conclusions of this work do not stand to be affected by overlooking a few weak detections of $\mathrm{CO}$ emission. The corresponding amounts of molecular hydrogen are small compared to the quantity of DNM, and small compared to the amounts of $\mathrm{H}_{2}$ that was inferred from the $\mathrm{HCO}^{+}$absorption.

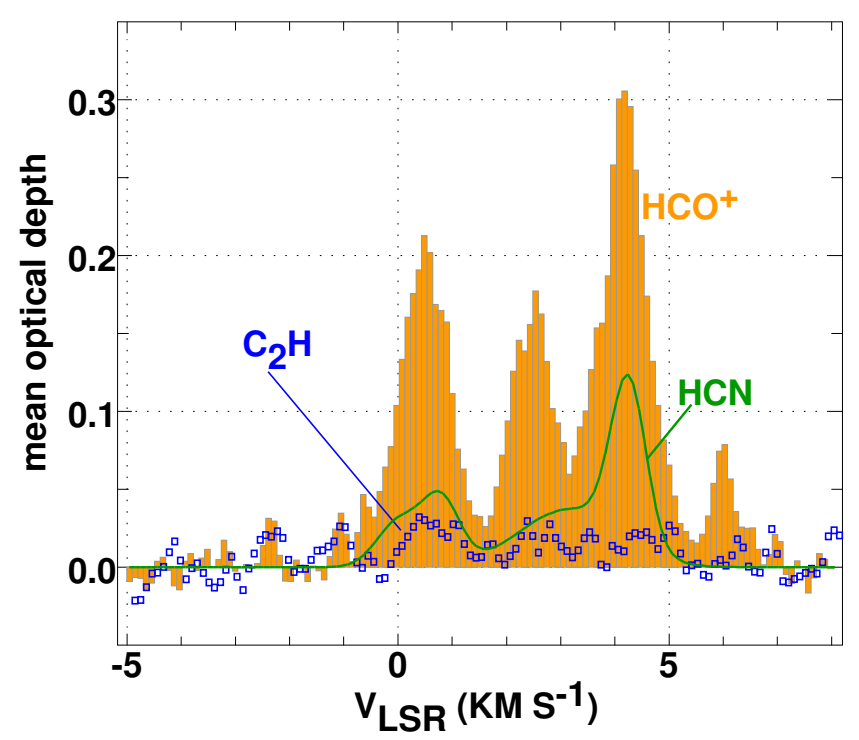

Fig. 5. Mean optical depth profiles for strongly polar molecules for the eight sources with $\mathrm{N}(\mathrm{DNM}) \gtrsim 2 \times 10^{20} \mathrm{~cm}^{-2}$. The $\mathrm{HCN}$ profile in green shows the kinematic structure that would be convolved with the HCN hyperfine splitting to reproduce the observations assuming that the hyperfine components appear in the LTE ratio 5:3:1. $\mathrm{C}_{2} \mathrm{H}$ is shown in blue.

The sightlines toward J1723 and J0942 have the highest molecular column density and would be expected to have the strongest $\mathrm{CO}$ emission. A typical global average for the conversion from $\mathrm{N}\left(\mathrm{H}_{2}\right)$ to $W_{\mathrm{CO}}$ is $X_{\mathrm{CO}}^{0}=2 \times 10^{20} \mathrm{H}_{2} \mathrm{~cm}^{-2}\left(\mathrm{~K}-\mathrm{km} \mathrm{s}^{-1}\right)^{-1}$ and the right-most column of Table 2 shows the predicted $\mathrm{CO}$ brightness derived from $\mathrm{N}\left(\mathrm{H}_{2}\right)$ as $W_{\mathrm{CO}}^{0}=\mathrm{N}\left(\mathrm{H}_{2}\right) / X_{\mathrm{CO}}^{0}$. The 
Table 3. Mean column densities according to sightline sample.

\begin{tabular}{lcccc}
\hline \hline Sample & $\langle\mathrm{N}(\mathrm{DNM})\rangle$ & $\left\langle 2 \mathrm{~N}\left(\mathrm{H}_{2}\right)\right\rangle$ & $\left\langle\left.\mathrm{N}(\mathrm{H} \mathrm{I})\right|_{\text {cham }}\right\rangle$ & $\left\langle 2 \mathrm{~N}\left(\mathrm{H}_{2}\right)\right\rangle /\langle\mathrm{N}(\mathrm{H})\rangle^{a}$ \\
\hline All & $2.1(1.9)$ & $3.8(3.7)$ & $6.2(1.8)$ & 0.38 \\
High DNM & $3.3(1.3)$ & $5.6(3.6)$ & $6.6(2.0)$ & 0.46 \\
Low DNM & $0.14(0.21)$ & $0.8(0.6)$ & $5.6(1.5)$ & 0.13 \\
\hline
\end{tabular}

Notes. All column densities are in units of $10^{20} \mathrm{~cm}^{-2}$. Quantities in parentheses are the standard deviation. ${ }^{(a)} \mathrm{N}(\mathrm{H})=\left.\mathrm{N}(\mathrm{H} \mathrm{I})\right|_{\text {cham }}+2 \mathrm{~N}\left(\mathrm{H}_{2}\right)$.

calculated values of $W_{\mathrm{CO}}^{0}$ for J1723 and J0942 from the entries in Table 2 are 2.8 and $2.0 \mathrm{~K}-\mathrm{km} \mathrm{s}^{-1}$, respectively (the observed value for $\mathrm{J} 1723$ is $2.4 \mathrm{~K}-\mathrm{km} \mathrm{s}^{-1}$ ), and there are three other cases in the higher-DNM group for which $W_{\mathrm{CO}}^{0} \gtrsim 1.5 \mathrm{~K}$. Thus, a CO survey with a robust detection limit of $1 \mathrm{~K}-\mathrm{km} \mathrm{s}^{-1}$ over an approximately $8 \mathrm{~km} \mathrm{~s}^{-1}$ velocity interval (the range observed here) might detect $\mathrm{CO}$ in a few other sightlines in the group with higher N(DNM).

\subsection{The $\mathrm{CO}-\mathrm{H}_{2}$ conversion factor}

A direct comparison of the measured $W_{\mathrm{CO}}$ and inferred $\mathrm{N}\left(\mathrm{H}_{2}\right)$ toward $\mathrm{J} 1723$ gives $\mathrm{N}\left(\mathrm{H}_{2}\right) / W_{\mathrm{CO}}=$ $2.3 \times 10^{20} \quad \mathrm{H}_{2} \quad \mathrm{~cm}^{-2}\left(\mathrm{~K}-\mathrm{km} \mathrm{s}^{-1}\right)^{-1}$ : the $\mathrm{HCO}^{+}$abundance chosen here quite generally yields $X_{\mathrm{CO}} \approx X_{\mathrm{CO}}^{0}$ in direct comparison between $\mathrm{HCO}^{+}$absorption and $\mathrm{CO}$ emission in galactic disk gas (Liszt et al. 2010; Gerin \& Liszt 2017). By contrast, studies like that of Planck Collaboration Int. XXVIII (2015) using dust and $\gamma$-ray or dust and X-ray (Lallement et al. 2016) measures of column density consistently find small values $X_{\mathrm{CO}} \lesssim 0.7 \times 10^{20} \mathrm{H}_{2} \quad \mathrm{~cm}^{-2}\left(\mathrm{~K}-\mathrm{km} \mathrm{s}^{-1}\right)^{-1}$ when averaged over whole structures or cloud complexes. If such small values of the $\mathrm{CO}-\mathrm{H}_{2}$ conversion factor were applied to the sightlines detected here in $\mathrm{HCO}^{+}$, the expected $W_{\mathrm{CO}}$ would have been three times higher than those in the right-most column of Table 2, and most of the sightlines in the higher DNM group would have shown detectable $\mathrm{CO}$ emission, some with $W_{\mathrm{CO}} \geq 5 \mathrm{~K}-\mathrm{km} \mathrm{s}^{-1}$. These were not seen by the NANTEN observations at $4^{\prime}$ spatial resolution and also are not present in the Planck Type 2 CO map at $6^{\prime}$ resolution.

For this reason we use $X_{\mathrm{CO}}=X_{\mathrm{CO}}^{0}$ for the weakly emitting diffuse molecular gas detected in the outskirts of Chamaeleon, while noting that smaller values apply in gas that is brighter in CO. Small regions of high CO brightness are in fact seen in the immediate vicinity of sightlines selected on the basis of their $\mathrm{HCO}^{+}$absorption when mapped in emission at $1^{\prime}$ resolution with the ARO KP $12 \mathrm{~m}$ antenna (Liszt \& Pety 2012). Moreover, the $\mathrm{CO}-\mathrm{H}_{2}$ conversion factors $\mathrm{N}\left(\mathrm{H}_{2}\right) / W_{\mathrm{CO}}$ averaged over the $\mathrm{CO}$ hotspots observed by Liszt \& Pety (2012) are smaller than $X_{\mathrm{CO}}^{0}$, as is expected for brighter $\mathrm{CO}$ emission from diffuse molecular gas (Liszt 2017).

\subsection{Molecular, missing, and CO-dark gas}

As shown in Table 2, somewhat more molecular gas was detected in total than was actually required by the DNM analysis of Planck Collaboration Int. XXVIII (2015). Figure 6 illustrates for the individual sightlines the manner in which the molecular gas absorption acts to make up the gas deficit. Along the horizontal axis is the total gas measure that is globally compared with and fit to the Fermi $\gamma$ ray emission and Planck dust optical depth maps to derive N(DNM), namely $\mathrm{N}(\mathrm{H})=\left.\mathrm{N}(\mathrm{H} \mathrm{I})\right|_{\text {cham }}+\mathrm{N}(\mathrm{DNM})+2 X_{\mathrm{CO}} W_{\mathrm{CO}}$ with $X_{\mathrm{CO}}=$ $X_{\mathrm{CO}}^{0}$ and $W_{\mathrm{CO}}=0$ for all sources except J1723. Along the
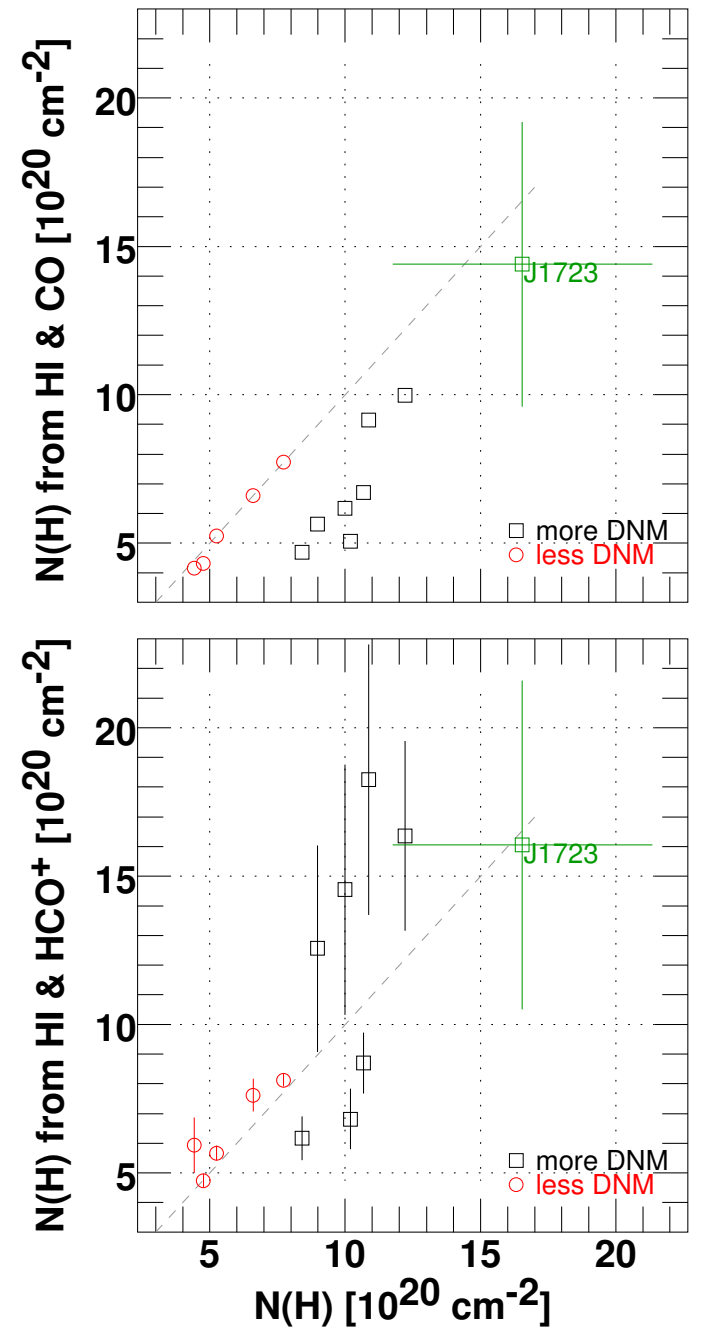

Fig. 6. Comparison of total column density of H-nuclei $\mathrm{N}(\mathrm{H})$ derived from the DNM analysis and from observable column densities. The quantity plotted along the horizontal axis, $\mathrm{N}(\mathrm{H})=\left.\mathrm{N}(\mathrm{H} \mathrm{I})\right|_{\text {cham }}+\mathrm{N}(\mathrm{DNM})+2 W_{\mathrm{CO}} X_{\mathrm{CO}}^{0}$, is the quantity fitted in the global DNM analysis and is the same in both panels. The vertical axis in each panel is the inferred total hydrogen column density $\mathrm{N}(\mathrm{H})=\left.\mathrm{N}(\mathrm{H} \mathrm{I})\right|_{\text {cham }}+2 \mathrm{~N}\left(\mathrm{H}_{2}\right)$ but calculated differently: at top, with $\mathrm{N}\left(\mathrm{H}_{2}\right)=W_{\mathrm{CO}} X_{\mathrm{CO}}^{0}$, and at bottom with $\mathrm{N}\left(\mathrm{H}_{2}\right)=\mathrm{N}\left(\mathrm{HCO}^{+}\right) / 3 \times 10^{-9}$. The error bars assume $\pm 50 \%$ errors in $W_{\mathrm{CO}}$ and $\mathrm{N}\left(\mathrm{HCO}^{+}\right) / \mathrm{N}\left(\mathrm{H}_{2}\right)$.

vertical axis we show two different versions of the total column density $\mathrm{N}(\mathrm{H})$ that is directly detectable in emission and/or absorption: at top is the combination of $\mathrm{N}\left(\mathrm{H}\right.$ I) and $\mathrm{H}_{2}$ determined from $\mathrm{CO}$ emission $\mathrm{N}(\mathrm{H})=\left.\mathrm{N}(\mathrm{H} \mathrm{I})\right|_{\text {cham }}+2 X_{\mathrm{CO}} W_{\mathrm{CO}}$ while the lower panel shows $\mathrm{N}(\mathrm{H})$ represented as the combination of $\mathrm{N}(\mathrm{H} \mathrm{I})$ with $\mathrm{H}_{2}$ determined in $\mathrm{HCO}^{+}$absorption, $\mathrm{N}(\mathrm{H})=\left.\mathrm{N}(\mathrm{H} \mathrm{I})\right|_{\text {cham }}+2 \mathrm{~W}_{\mathrm{HCO}^{+}} / 3 \times 10^{-9}$. The error bars assume $\pm 50 \%$ errors in $W_{\mathrm{CO}}$ and $\mathrm{N}\left(\mathrm{HCO}^{+}\right) / \mathrm{N}\left(\mathrm{H}_{2}\right)$. 
In Fig. 6, the five sightlines with small N(DNM) are on the equality line in the upper panel when the $\mathrm{CO}$ emission is used, and they remain near it when $\mathrm{H}_{2}$ is derived from $\mathrm{HCO}^{+}$because they intercept mostly atomic gas with only very weak molecular absorption. The sightline toward J1723 is near the equality line when $\mathrm{CO}$ is used and somewhat closer to it when $\mathrm{N}\left(\mathrm{H}_{2}\right)$ is derived from $\mathrm{HCO}^{+}$for reasons that are discussed in Sect. 3.5. The remaining seven sightlines lie well below the equality line when $\mathrm{N}\left(\mathrm{H}_{2}\right)$ is derived from $\mathrm{CO}$ emission in the upper panel and bracket it when $\mathrm{HCO}^{+}$is used.

\subsection{Saturation of the $\lambda 21 \mathrm{~cm}$ emission}

Fukui et al. (2015) and Okamoto et al. (2017) have argued that $\mathrm{CO}$ emission does not miss any substantial amount of molecular gas and that high optical depth and saturation of the $\lambda 21 \mathrm{~cm} \mathrm{H}$ I profile can account for the DNM that is seen in analyses like that of Planck Collaboration Int. XXVIII (2015) and Remy et al. (2018). Given the pervasive molecular absorption discovered in this work, it seems difficult to support any argument that dismisses the importance of diffuse molecular gas that is missed in $\mathrm{CO}$ emission. However, the possible saturation of $\mathrm{H} \mathrm{I}$ emission on some lines of sight is a somewhat separate issue. The DNM analysis was performed for a series of uniform gas temperatures ranging from 125 to $800 \mathrm{~K}$, and it was concluded that the optically thin limit provided the best overall fit to interpret $\mathrm{H} \mathrm{I}$ emission. However, it is possible that the amount of atomic gas is underestimated in localised regions.

With reference to Table 2, there is a shortfall of molecular gas, $2 \mathrm{~N}\left(\mathrm{H}_{2}\right)<\mathrm{N}(\mathrm{DNM}) / 2$, for three of the four sources having the highest DNM values. These are the sightlines at intermediate $\mathrm{N}(\mathrm{H})$ that fall below the equality line in the lower panel of Fig. 6 and two of them have quite small values of $\mathrm{N}(\mathrm{H}) / E_{B-V}$ in Table 2. Our results show that the loss of gas due to saturation of the $\mathrm{H}$ I line profile is small overall because the amount of gas that would need to be added to those sightlines to bring their $\mathrm{N}(\mathrm{H})$ or $\mathrm{N}(\mathrm{H}) / E_{B-V}$ in line with the other sources is a small fraction of the total amount of gas sampled. That said, saturation of the $\mathrm{H}$ I profile may occur along three of the sightlines studied here, and $\mathrm{H} \mathrm{I}$ absorption measurements in those directions might shed some light on this issue.

\section{Summary}

Using the ALMA telescope we searched for and detected $89.2 \mathrm{GHz} \mathrm{HCO}^{+}$absorption in 13 directions lying toward the outskirts of the Chamaeleon cloud complex as summarized in Table 1 and illustrated on the sky in Fig. 1. The directions were selected to include the known mm-wave calibrator sources with fluxes above $125 \mathrm{mJy}$ in March 2016 when the observing proposal was written, and a larger complement of strong calibrators has not been forthcoming in the intervening time. $\mathrm{CO}$ emission and $\mathrm{HCO}^{+}$absorption profiles are compared in Fig. 2: $\mathrm{CO}$ emission had been firmly detected in only one of these directions, toward the source $\mathrm{J} 1723$. Weak $\mathrm{C}_{2} \mathrm{H}$ absorption was detected in eight directions and $\mathrm{HCN}$ emission in four directions.

As shown in Table 2, the sightlines fall into two groups having eight and five members, depending on whether the column density of dark neutral medium N(NDM) $\gtrsim 2.0 \times 10^{20} \mathrm{~cm}^{-2}$ or $\mathrm{N}(\mathrm{DNM})<0.5 \times 10^{20} \mathrm{~cm}^{-2}$, respectively. The two groups have similar mean total $\mathrm{H}$ I column density $\left\langle\left.\mathrm{N}(\mathrm{H} \mathrm{I})\right|_{\text {tot }}\right\rangle=13 \times$ $10^{20} \mathrm{~cm}^{-2}$, comparable N(H I) associated with the Chamaeleon complex $\left\langle\left.\mathrm{N}(\mathrm{H} \mathrm{I})\right|_{\text {cham }}\right\rangle=5.6-6.6 \times 10^{20} \mathrm{~cm}^{-2}$ (smaller in the low-DNM group) and mean total column density per unit reddening $\left\langle\mathrm{N}(\mathrm{H}) / E_{B-V}\right\rangle \approx 5.4-6.7 \times 10^{21} \mathrm{~cm}^{-2}(\mathrm{mag})^{-1}$. By contrast, the group of eight sightlines with more DNM has much larger mean reddening $\left\langle E_{B-V}\right\rangle=0.33$ vs. $0.18 \mathrm{mag}$, mean DNM column density $\langle\mathrm{N}(\mathrm{DNM})\rangle=3.3$ vs. $0.14 \times$ $10^{20} \mathrm{~cm}^{-2}$ and mean column density of H-nuclei in molecular form $\left\langle 2 \mathrm{~N}\left(\mathrm{H}_{2}\right)\right\rangle=5.6$ vs. $0.83 \times 10^{20} \mathrm{~cm}^{-2}$.

As shown in Figs. 3-5 and discussed in Sect. 3.2, there is a kinematic signature to the difference between the two groups, as the group with higher N(DNM) has stronger H I emission at $2 \mathrm{~km} \mathrm{~s}^{-1} \lesssim v \lesssim 8 \mathrm{~km} \mathrm{~s}^{-1}$, where the bulk of the molecular absorption also occurs. Molecular absorption in the group having smaller $\mathrm{N}(\mathrm{DNM})$ is confined to velocities $v \lesssim 2 \mathrm{~km} \mathrm{~s}^{-1}$ even though the sightlines comprising the group span the entire breadth of the cloud complex (Fig. 1) and in principle could have sampled the full range of velocities. Molecule formation in the group with higher DNM may have been enhanced by slow shocks associated with turbulence.

On the whole, somewhat more $\mathrm{H}_{2}$ was detected in $\mathrm{HCO}^{+}$ than previously found in the DNM analysis (Table 2, Sect. 3.3) but the neutral gas in the outskirts of Chamaeleon is mostly atomic. $\mathrm{H}_{2}$ bears $46 \%$ of the $\mathrm{H}$-nuclei in the group having higher $\mathrm{N}(\mathrm{DNM}), 13 \%$ in the gas having lower N(DNM) and $36 \%$ in total. This is within the range of estimates of the molecular gas fraction in the diffuse ISM as a whole as noted in Sect. 3.3. There is no reservoir of "dark" molecular gas that went undetected as part of the inventory of dark neutral medium. Saturation of the $\mathrm{H}$ I profile may have caused some atomic gas to be missed along 3 of the 13 sightlines observed here (Sect. 3.6), and observations of $\mathrm{HI}$ absorption might help to understand why the molecular gas column density was smaller than that of the inferred DNM in those directions. Nonetheless, DNM is overwhelmingly in the form of molecular gas overall.

Thus the situation is clear for sightlines with weak or absent $\mathrm{CO}$ emission in the outskirts of the Chamaeleon complex: the DNM is primarily molecular gas that was missed in $\mathrm{CO}$ emission and to a much lesser extent, atomic gas that might have been missed at $\lambda 21 \mathrm{~cm}$. We note, however, that the present group of mm-wave bright quasars did not sample sightlines with twicelarger DNM colum densities that are present around the main Cha I and Cha II + III clouds. Probing the dense HI and diffuse $\mathrm{H}_{2}$ composition of the DNM in those directions would be valuable. DNM was also detected toward regions with high $\mathrm{CO}$ intensities $W_{\mathrm{CO}}>7 \mathrm{~K}-\mathrm{km} \mathrm{s}^{-1}$, which was treated as an independent component in the gamma-ray analysis of Remy et al. (2018) as it likely captures additional molecular gas in which emission saturates in the main $\mathrm{CO}$ isotopologue and ${ }^{13} \mathrm{CO}$ or $\mathrm{C}^{18} \mathrm{O}$ should be used. Taking the molecular gas census in those directions would also shed important light on $X_{\mathrm{CO}}$ gradients across molecular clouds.

Acknowledgements. This paper makes use of the following ALMA data: ADS/JAO.ALMA\#2016.1.00714.S. ALMA is a partnership of ESO (representing its member states), NSF (USA) and NINS (Japan), together with NRC (Canada), NSC and ASIAA (Taiwan), and KASI (Republic of Korea), in cooperation with the Republic of Chile. The Joint ALMA Observatory is operated by ESO, AUI/NRAO and NAOJ. The National Radio Astronomy Observatory is a facility of the National Science Foundation operated under cooperative agreement by Associated Universities, Inc. This work was supported by the French program Physique et Chimie du Milieu Interstellaire (PCMI) funded by the Conseil National de la Recherche Scientifique (CNRS) and Centre Nationa d'Etudes Spatiales (CNES). HSL is grateful to the hospitality of the ITU-R and the Hotel Bel Esperance in Geneva during the completion of this manuscript, and the incomparable Hotel Ritz in Madrid. We thank Sarah Wood, Devaky Kunneriath and the data analysts at the North American ALMA Science Center for producing the imaging scripts for this project and sheparding it through the data reduction process. We thank the anonymous referee for comments. 
H. Liszt et al.: Molecular gas and dark neutral medium in the outskirts of Chamaeleon

\section{References}

Ando, R., Kohno, K., Tamura, Y., et al. 2016, PASJ, 68, 6 Burgh, E. B., France, K., \& McCandliss, S. R. 2007, ApJ, 658, 446

Clark, B. G. 1965, ApJ, 142, 1398

Donate, E., \& Magnani, L. 2017, MNRAS, 472, 3169

Fukui, Y., Torii, K., Onishi, T., et al. 2015, ApJ, 798, 6

Gerin, M., \& Liszt, H. 2017, A\&A, 600, A48

Grenier, I. A., Casandjian, J.-M., \& Terrier, R. 2005, Science, 307, 1292

Kalberla, P. M. W., \& Haud, U. 2015, A\&A, 578, A78

Lallement, R., Snowden, S., Kuntz, K. D., et al. 2016, A\&A, 595, A131

Liszt, H. S. 2012, A\&A, 538, A27

Liszt, H. S. 2017, ApJ, 835, 138

Liszt, H. S., \& Gerin, M. 2016, A\&A, 585, A80

Liszt, H., \& Gerin, M. 2018, A\&A, 610, A49

Liszt, H. S., \& Lucas, R. 1996, A\&A, 314, 917

Liszt, H. S., \& Lucas, R. 1998, A\&A, 339, 561

Liszt, H., \& Lucas, R. 2001, A\&A, 370, 576

Liszt, H., \& Lucas, R. 2002, A\&A, 391, 693

Liszt, H. S., \& Pety, J. 2012, A\&A, 541, A58

Liszt, H. S., \& Pety, J. 2016, ApJ, 823, 124

Liszt, H. S., Pety, J., \& Lucas, R. 2010, A\&A, 518, A45
Lucas, R., \& Liszt, H. S. 1996, A\&A, 307, 237

Lucas, R., \& Liszt, H. S. 2000, A\&A, 358, 1069

Micic, M., Glover, S. C. O., Federrath, C., \& Klessen, R. S. 2012, MNRAS, 421, 2531

Mizuno, A., Yamaguchi, R., Tachihara, K., et al. 2001, Publ. Astron. Soc. Jpn., 53, 1071

Okamoto, R., Yamamoto, H., Tachihara, K., et al. 2017, ApJ, 838, 132

Planck Collaboration XI. 2014, A\&A, 571, A11

Planck Collaboration Int. XXVIII. 2015, A\&A, 582, A31

Remy, Q., Grenier, I. A., Marshall, D. J., \& Casandjian, J. M. 2018, A\&A, 611, A51

Riquelme, D., Bronfman, L., Mauersberger, R., et al. 2018, A\&A, 610, A43

Savage, B. D., Drake, J. F., Budich, W., \& Bohlin, R. C. 1977, ApJ, 216, 291

Schlegel, D. J., Finkbeiner, D. P., \& Davis, M. 1998, ApJ, 500, 525

Sheffer, Y., Rogers, M., Federman, S. R., et al. 2008, ApJ, 687, 1075

Sonnentrucker, P., Welty, D. E., Thorburn, J. A., \& York, D. G. 2007, ApJS, 168, 58

Valdivia, V., Hennebelle, P., Godard, B., et al. 2017, ArXiv e-prints [arXiv: 1711.01824]

Weselak, T., Galazutdinov, G. A., Beletsky, Y., \& Krełowski, J. 2010, MNRAS, 402, 1991

Wolfire, M. G., Hollenbach, D., \& McKee, C. F. 2010, ApJ, 716, 1191 